\title{
Spontaneous Conception after Hysterosalpingography in an Infertile Woman with Bilateral Tubal Factors: A Case Report
}

\author{
Ayissi Grégory ${ }^{1,2 *}$, Astrid Ndolo Kondo', Kasia Onana Yves Bertrand ${ }^{1}$, Ebode Koa \\ Vanessa $^{1}$, Azambou Kenteu Sandrine ${ }^{1}$, Ngo Dingom Anne Madye ${ }^{2}$ and Essiben Felix ${ }^{1,2}$ \\ ${ }^{1}$ Department of Gynecology and Obstetrics, Faculty of Medicine and Biomedical Science, University of \\ Yaounde I, Yaoundé, Cameroon
}

${ }^{2}$ Principal Maternity, Yaounde Central Hospital, Cameroon

*Corresponding author: Ayissi Grégory, Department of Gynecology and Obstetrics, Faculty of Medicine and Biomedical Science, University of Yaounde I, 5008, Yaoundé, Cameroon

\begin{abstract}
Background: Hysterosalpingography (HSG) is an outpatient fluoroscopy procedure that evaluates the uterine cavity and fallopian tube patency. In some cases, HSG may have a therapeutic effect.

Case presentation: We present the case of a 35-year-old HIV-positive woman with a 3-years-old history of secondary tubal infertility, a hysterosalpingogram revealing bilateral proximal tubal obstruction and presented later on with a 6-weeks pregnancy on ultrasound after HSG evaluation.

Conclusion: Hysterosalpingography (HSG) is an inexpensive and widely available test to assess tubal infertility. Its therapeutic effects, although rare, can lead to pregnancy in infertile women.
\end{abstract}

\section{Keywords}

Hysterosalpingography, Infertility, Tubal factors, Spontaneous conception

\section{Introduction}

HSG is the evaluation of the uterine cavity, fallopian tubes, and adjacent peritoneal cavity following the injection of contrast material through the cervical canalunder fluoroscopy [1].

The primary role of Hysterosalpingography (HSG) is to evaluate the morphology and the patency of the fallopian tubes in female infertility, but it remains a major diagnosis tool in assessing intrauterine abnormalities [2]. In tubal pathology, findings mainly include tubal ob- struction, spasm and hydrosalpinx while others lesions are peritubal adhesions, salpingitis isthmica nodosa and genital track tuberculosis [1].

Several factors affect HSG test performance such as underlying pathology, the training and experience of the clinician(s) performing the procedure and interpreting the images, and the standard used to validate the HSG results. HSG is most useful for predicting tubal occlusion rather than uterine pathology [1]. For example, In a meta-analysis of nine studies comparing HSG with laparoscopy as the standard, the pooled estimates of sensitivity and specificity for HSG in identifying tubal occlusion were 0.94 (95\% Cl 0.47-0.99) and 0.92 (95\% $\mathrm{Cl}$ 0.87-0.95), respectively [3]. In contrast, a prospective study of 336 women undergoing both HSG and diagnostic hysteroscopy (reference standard), reported the ability of HSG to detect intrauterine pathology with sensitivity and specificity of 98 and 35\% respectively [4].

Tubal patency test including HSG and Hystero-contrast-sonography (HyCoSy) have been associated with spontaneous pregnancy in the literature. For example, the spontaneous pregnancy rate after HyCoSy was $19.44 \%$ within 180 days in a prospective study about 1008 infertile Chinese women [5]. The main theory underpinning this hypothesis is that the passage of liquid breaks minor adhesions or flushes mucus plugs through the tubes.

In our observation, this was the first case of sponta- 


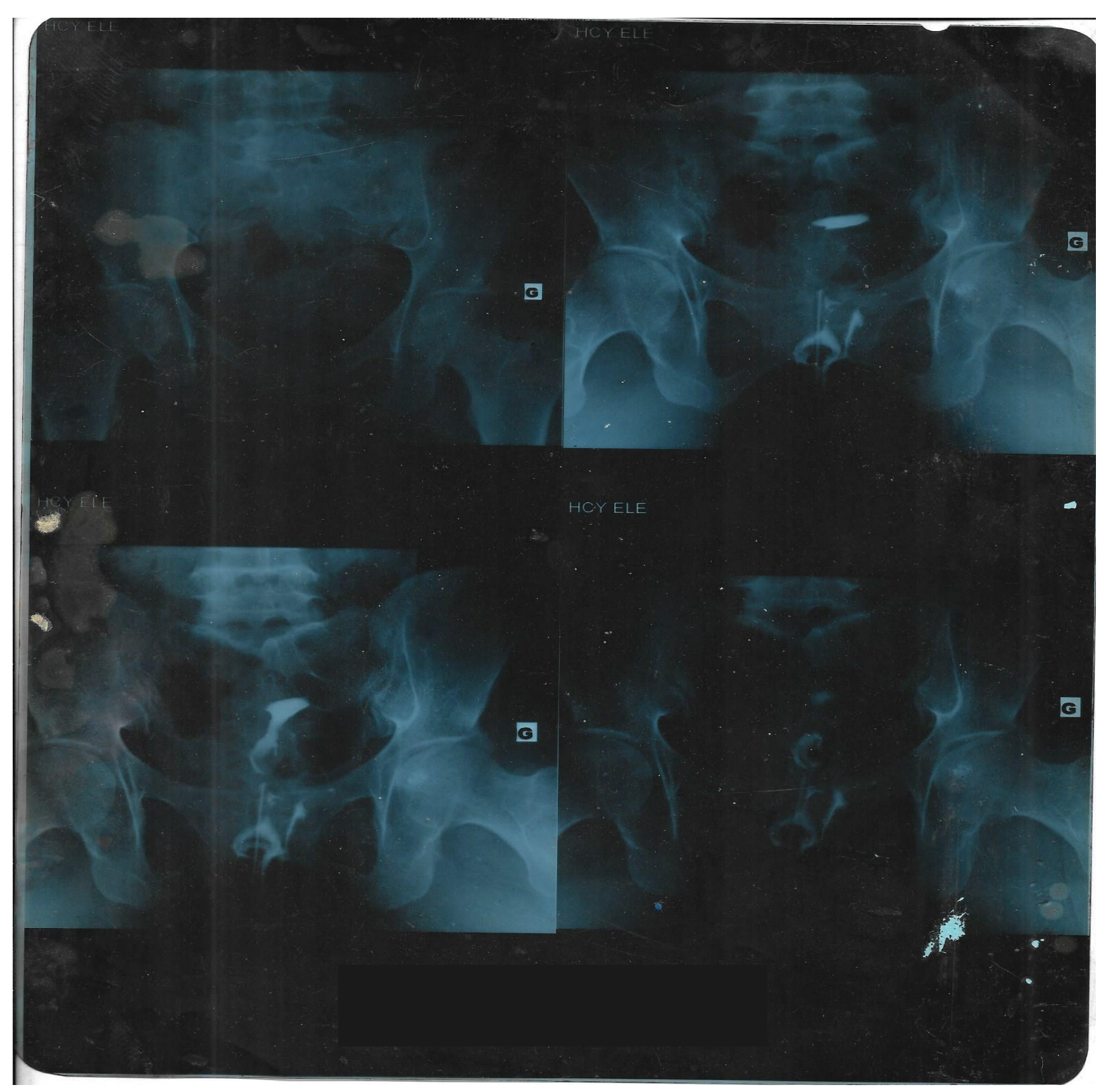

Figure 1: Bilateral proximal obstruction on HSG.

neous pregnancy after HSG in an infertile woman, and we sought to discuss factors that led to conception in a clinical case report.

\section{Case Presentation}

We present the case of a 35-years-old, HIV-positive known since 6 years, in the course of her first pregnancy, undergoing antiretroviral treatment. She had her last menses on the 29/08/19 and a history of two spontaneous pregnancies with the same partner resulting in full-term vaginal deliveries, including one stillborn due to intrapartum asphyxia. She attended our outpatients unit for a desire to conceive evolving since 3 years. The initial clinical examination was without particularities. The current infectious balance sheet revealed Ureaplasma urealyticum infection and Gardnerella vaginitis; she was treated with antibiotics including Doxycycline and
Tinidazol. A pelvic ultrasound revealed signs of Bilateral adnexitis (bilateral utero-ovarian adhesion), a fine and regular endometrium, and a low abundance fluid effusion in the Douglass. Her partner was undergoing antiretroviral therapy for HIV since 6 years and had a normal infectious balance sheet and a normal sperm count.

The patient came back two months later with a recurrent pelvic mycoplasma infection treated with Ofloxacin. A post-antibiotic hysterosalpingography requested for tubal assessment on 05/09/19 revealed a bilateral proximal tubal obstruction (Figure 1). Then on $31 / 10 / 19$, she came back with an ultrasound showing a 6-week pregnancy alongside a 35\% retro-trophoblastic fundic hematoma (Figure 2). The patient was placed on progesterone, folic acid and oral iron, and the rest of the pregnancy's follow-up was uneventful. 

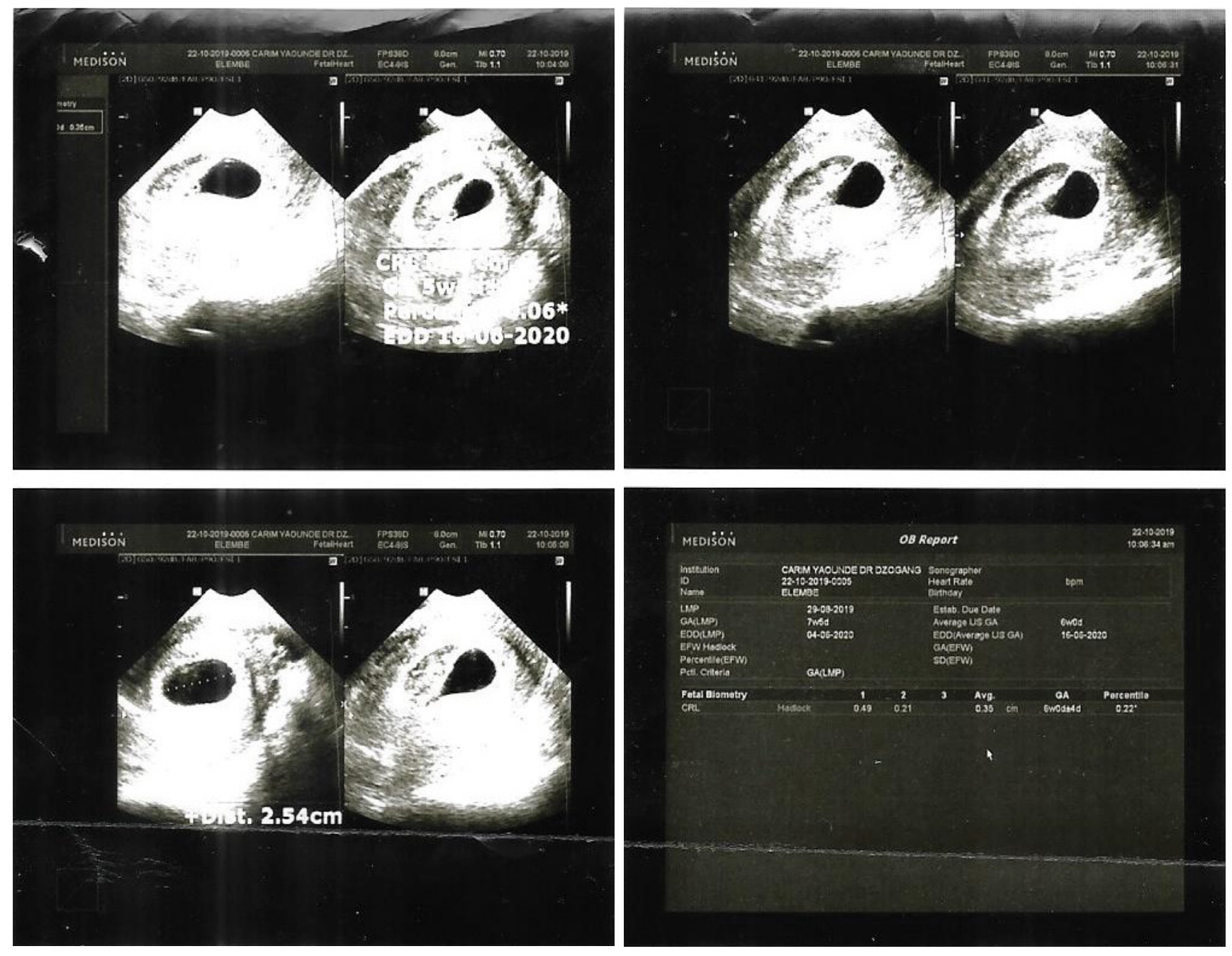

Figure 2: Ultrasound image of 6 week pregnancy and retro-trophobalstic hematoma.

\section{Discussion}

Age, HIV and Mycoplasm infections were the major risk factors for tubal occlusion in our patient. Indeed, demographic characteristics that have been reported to be associated with low rates of pregnancy among HIV-infected women include advanced reproductive age, with the subsequent tubal damage of others Sexual transmitted infections (STIS) associated with HIV [6]. The impact of STIs on fertility among person living with HIV (PLWHIV) depends on the local prevalence of the STIs. In our setting this prevalence is 5.2\% [7]. Given the immunosuppressive status caused by HIV, STIs tend to be more prevalent, more severe, to take longer to resolve, and to be more prone to treatment failure in HIV-infected persons compared to the general population. The underlying pathogenesis for STI-related infertility is direct damage and subsequent anatomical and functional abnormalities of the genital organs including cervix, endometrium, fallopian tubes and ovaries; leading to tubal occlusions and ectopic pregnancies [8].

Spontaneous pregnancy over a tubal obstruction may be considered providential, but several factors could explain it. Since the interpretation of HSG is operator-dependent, a cornual spasm in our case could have been considered a tubal obstruction. Indeed, muscle spasm can cause transient tubal occlusion and prevent contrast from filling an otherwise patent tube. Spasm is characterized by a rounded smooth cornual margin, whereas cornual occlusion is characterized by pointed or irregular cornual margin, although these changes can be difficult to distinguish definitively [1]. Repeating the examination at a future date typically allows differentiation of the tube in spasm from one that is permanently obstructed. Note, a historical report described administering $1 \mathrm{mg}$ of glucagon intravenously to cause muscle relaxation and thus enables contrast flow and tube opacification, but this approach is not a common practice [1].

Aside the infection sequelae, the tubal obstruction in our case could be due to salpingitis isthmica nodosa which is an acquired condition in which mucosal glands penetrate the myosalpinx with resultant hyperplasia and hypertrophy [1]. The characteristic appearance on HSG is that of multiple, small diverticula extending from the isthmic lumen into the wall, which is sometimes, described as tubal diverticulosis. This condition affects both tubes in $80 \%$ of cases and is frequently associated with ampullary dilatation or obstruction proximally [9]. The condition is strongly associated with infertility 
and ectopic pregnancy but in some rare cases the tubes could remain patent and spontaneous pregnancy can occur [10].

The therapeutic effects of tubal patency test are widely discussed in the literature and HSG is not an exception to that [11]. In women with tubal obstruction, the passage of the contrast medium can break minor adhesions or flushes mucus plugs through the tubes, leading to tubal repermeabilization and spontaneous pregnancy as it could be in our case. In this woman, the HSG was performed with iodinated water-soluble contrast media. Indeed, the role of contrast medium nature in the occurrence of spontaneous pregnancy after hysterosalpingography is well discussed. As example, evaluating outcomes after the use of water-soluble contrast media versus oil-based contrast media in 1100 infertile women, Spring, et al. found no difference in the two groups, in term of spontaneous pregnancies [12]. In the same vein, a smaller randomized trial and a meta-analysis of this trial plus one other that included infertile women at high risk for tubal disease did not report a higher life birth rate with oil-based contrast [13].

But water-soluble contrast remains the main media used, because it provides higher quality images and does not require precautionary measures to avoid the small risk of pulmonary and cerebral embolism that can occur with intravascular injection of oil-based contrast [1].

Thus HSG is a cheap, accessible patency test which can lead to spontaneous pregnancy in women with bilateral tubal factors infertility but remains risky in term of false positive (operator dependant character), radiation exposure, iodine allergic reactions and infections.

\section{Conclusion}

Hysterosalpingography (HSG) is an inexpensive and widely available test to assess tubal infertility. Its therapeutic effects, although rare, can lead to pregnancy in infertile women.

\section{References}

1. Lee IL, A Kilcoyne (2019) Hysterosalpingography. UpToDate. 2019.

2. Schankath AC, Fasching N, Urech-Ruh C, Hohl MK, Kubik-Huch RA (2012) Hysterosalpingography in the workup of female infertility: Indications, technique and diagnostic findings. Insights Imaging 3: 475-483.

3. Maheux-Lacroix S, Boutin A, Moore L, Bergeron M-E, Bujold $E$, et al. (2014) Hysterosalpingosonography for diagnosing tubal occlusion in subfertile women: A systematic review with meta-analysis. Hum Reprod 29: 953-963.

4. Preutthipan S, Linasmita V (2003) A prospective comparative study between hysterosalpingography and hysteroscopy in the detection of intrauterine pathology in patients with infertility. J Obstet Gynaecol Res 29: 33-37.

5. Chunyan G, Bin P, Ping Y, Yue Z, Yang X, et al. (2018) Assessment of the influence on spontaneous pregnancy of hysterosalpingo-contrast sonography. Biomed Res Int.

6. Khawcharoenporn T, Sha BE (2016) HIV Infection and Infertility. Genital Infections and Infertility.

7. Nkwabong E, Dingom MAN (2015) Acute pelvic inflammatory disease in cameroon: A cross sectional descriptive study. Afr J Reprod Health 19: 87-91.

8. Etienne B, Claude Cyrille NN, Gregory A, Yves Bertrand KO, Jean-Marie K (2018) Laparoscopic management of an ectopic pregnancy after in vitro fertilization and embryo transfer at CHRACERH: A case report. Laparosc Endosc Robot Surg 1: 70-73.

9. Suresh YN, Narvekar NN (2014) The role of tubal patency tests and tubal surgery in the era of assisted reproductive techniques. Obstet Gynaecol 16: 37-45.

10. Kurtoglu E, Kokcu A, Celik H, Kefeli M (2015) Salpingitis isthmica nodosa and recurrent ectopic pregnancy. J Obstet Gynaecol 35: 217-218.

11. Giugliano E, Cagnazzo E, Bazzan E, Patella A, Marci R (2012) Hysterosalpingo-contrast sonography: Is possible to quantify the therapeutic effect of a diagnostic test? Clin Exp Reprod Med 39: 161-165.

12. Spring DB, Barkan HE, Pruyn SC (2000) Potential therapeutic effects of contrast materials in hysterosalpingography: A prospective randomized clinical trial. Radiology 214 : 53-57.

13. Mohiyiddeen L, Hardiman A, Fitzgerald C, Hughes E, Mol BWJ, et al. (2015) Tubal flushing for subfertility. Cochrane Database Syst Rev. 